\title{
OPCML Gene
}

National Cancer Institute

\section{Source}

National Cancer Institute. OPCML Gene. NCI Thesaurus. Code C116960.

This gene is involved in opioid binding. 